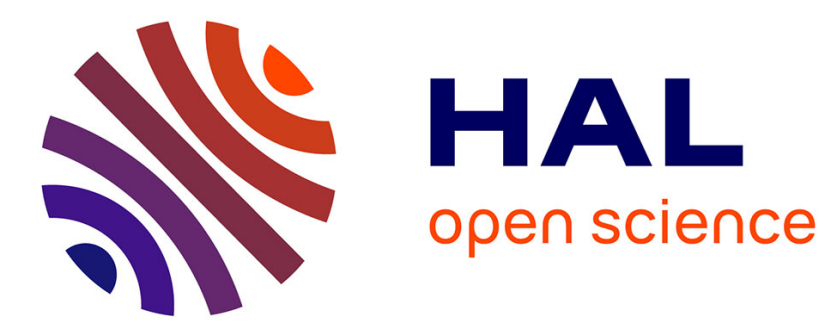

\title{
Observability and synchronization of neuron models
}

\author{
Luis A Aguirre, Leonardo L. Portes, Christophe Letellier
}

\section{To cite this version:}

Luis A Aguirre, Leonardo L. Portes, Christophe Letellier. Observability and synchronization of neuron models. Chaos: An Interdisciplinary Journal of Nonlinear Science, 2017, 27 (10), 10.1063/1.4985291. hal-01672077

HAL Id: hal-01672077

https://hal-normandie-univ.archives-ouvertes.fr/hal-01672077

Submitted on 23 Dec 2017

HAL is a multi-disciplinary open access archive for the deposit and dissemination of scientific research documents, whether they are published or not. The documents may come from teaching and research institutions in France or abroad, or from public or private research centers.
L'archive ouverte pluridisciplinaire HAL, est destinée au dépôt et à la diffusion de documents scientifiques de niveau recherche, publiés ou non, émanant des établissements d'enseignement et de recherche français ou étrangers, des laboratoires publics ou privés. 


\section{Observability and synchronization of neuron models}

Luis A. Aguirre, Leonardo L. Portes, and Christophe Letellier

Citation: Chaos 27, 103103 (2017); doi: 10.1063/1.4985291

View online: http://dx.doi.org/10.1063/1.4985291

View Table of Contents: http://aip.scitation.org/toc/cha/27/10

Published by the American Institute of Physics 


\title{
Observability and synchronization of neuron models
}

\author{
Luis A. Aguirre, ${ }^{1,2, a)}$ Leonardo L. Portes, ${ }^{2}$ and Christophe Letellier ${ }^{3}$ \\ ${ }^{1}$ Departamento de Engenharia Eletrônica, Universidade Federal de Minas Gerais, Belo Horizonte \\ 31.270-901, Minas Gerais, Brazil \\ ${ }^{2}$ Programa de Pós-Graduação em Engenharia Elétrica da Universidade Federal de Minas Gerais-Av. \\ Antônio Carlos 6627, 31.270-901 Belo Horizonte, Minas Gerais, Brazil \\ ${ }^{3}$ CORIA-UMR 6614, Normandie Université, Campus Universitaire du Madrillet, F-76800 Saint-Etienne du \\ Rouvray, France
}

(Received 29 May 2017; accepted 19 September 2017; published online 3 October 2017)

\begin{abstract}
Observability is the property that enables recovering the state of a dynamical system from a reduced number of measured variables. In high-dimensional systems, it is therefore important to make sure that the variable recorded to perform the analysis conveys good observability of the system dynamics. The observability of a network of neuron models depends nontrivially on the observability of the node dynamics and on the topology of the network. The aim of this paper is twofold. First, to perform a study of observability using four well-known neuron models by computing three different observability coefficients. This not only clarifies observability properties of the models but also shows the limitations of applicability of each type of coefficients in the context of such models. Second, to study the emergence of phase synchronization in networks composed of neuron models. This is done performing multivariate singular spectrum analysis which, to the best of the authors' knowledge, has not been used in the context of networks of neuron models. It is shown that it is possible to detect phase synchronization: (i) without having to measure all the state variables, but only one (that provides greatest observability) from each node and (ii) without having to estimate the phase. Published by AIP Publishing. https://doi.org/10.1063/1.4985291
\end{abstract}

The state of a neuron model can be trivially reconstructed if all variables are measured. In practice that is not the case. If only one variable can be measured, which would be the most adequate one to reconstruct the phase space? The observability theory provides answers to this question. Although such a theory has been developed and applied to a number of systems in nonlinear dynamics, it does face some new challenges when applied to some neuron models. This paper describes results of three different observability measures applied to four neuron models commonly used in the literature. Given a network composed of neuron models, it is often desirable to be able to detect phase synchronization (PS). This situation has a number of implications and applications in neuroscience. A second aim of this paper is to provide numerical evidences that structured-varimax multivariate singular spectrum analysis (svM-SSA) of network data can be used to detect phase synchronization, without having to estimate phases, by processing one variable from each node of the network. The use of a single variable is viable as long as the used variable is one that provides good observability of the dynamics compared to the rest.

\section{INTRODUCTION}

Since the early days of the last century, there has been sustained activity in developing mathematical models for neuron dynamics. More recently, such models have been combined in networks in order to investigate collective

\footnotetext{
${ }^{a)}$ Electronic mail: aguirre@ufmg.br
}

behavior. In either approaches mathematical tools and concepts abound. The authors of Ref. 42 argue that there must be a continued effort in using such models to reveal so many aspects of the brain dynamics which remain ununderstood. The same point had been argued by Brown in a very entertaining discussion. ${ }^{8}$

In this respect, an important concept is that of observability of the dynamics from a given measured variable. Since it is not practical, especially in high-dimension systems, to record all state variables, a relevant problem is to know which are the best variables to record to be able to infer the state of the whole system. Observability, although not in its classical interpretation, provides an answer to that question. It has been acknowledged that to choose variables that provide good observability of the dynamics enables estimating the state of a network of neuron models using Kalman-related methods. ${ }^{39,40}$ In a recent study about controllability and observability of network motifs built with neuron models, it has been found that "it is necessary to take the node dynamics into consideration when selecting the best driver (sensor) node to modulate (observe) the whole network activity" Ref. 43 (Sec. III A).

In view of this, one of the aims of this paper is to conduct a study of observability properties of four neuron models following three points of view: using the model equations and numerical analysis, ${ }^{29}$ using the model equations and symbolic manipulations, ${ }^{28}$ and using time series data. ${ }^{3} \mathrm{An}$ interesting point that has been revealed in this study is related to aspects that are specific to neuron models. For instance, in the case of the Hodgkin-Huxley model, three of the four state variables are not directly measurable. The 
study of observability could help understand if there are any serious limitations related to this. In such a case, the use of observability coefficients estimated from data is most convenient, because the practical relevance of measuring ionic currents could be evaluated. Due to the functional relation of such currents with the state variables, the computation of observability coefficients from the equations is significantly more difficult. Other examples are the integrate and fire models, that produce discontinuities in the data. Such a phenomenon may have adverse effects on data-driven observability coefficients, and the equationbased computation of coefficients is also questionable because of the "hidden state variable" related to the firing process. These aspects, that have come to light in the context of the investigated neuron models, are here described for the first time.

Another important aspect that has gained considerable attention is that of synchronization of networked neuron models. Because in real life neurons are not identical and coupling could be weak, phase synchronization (PS) is somewhat more well suited than complete synchronization in this context. A difficulty with most procedures used to detect PS is the need for defining a phase, which is not always simple, if at all possible. Spectral coherence related measures of PS have recently been considered and found to deviate considerably from expected results. ${ }^{32}$ An alternative procedure that does not require the estimation of the phase is the multivariate singular spectrum analysis (M-SSA) for PS phenomena, originally proposed by Groth and Ghil. ${ }^{14}$ This method will be reviewed and applied to detect PS in networks of neuron models in this work for the first time, to the best of the authors' knowledge. Although observability and synchronizability are different problems and treated as such in this paper, there is a connection between them in the context of multivariate singular spectrum analysis, as will be pointed out.

This paper is organized as follows. For the sake of completion, there are two sections with the background material. Section II briefly describes four of the neuron models considered in this study. Section III reviews the main tools used. The numerical results are presented in Sec. III D and the results regarding synchronization are briefly described in Sec. IV. Conclusions are provided in Sec. V.

\section{NEURON MODELS}

Four neuron models that will be used are presented. 2D, 3D, and 4D models and a switching model of the type integrate and fire were chosen. It is believed that this choice is representative but admittedly it is also somewhat arbitrary. For a general comparison of the models, see Izikevich's review. ${ }^{21}$

\section{A. The Hodgkin-Huxley model}

The Hodgkin-Huxley, a biophysically-based model of neuron dynamics, is given by ${ }^{18}$

$$
\left\{\begin{array}{l}
\dot{V}=\frac{1}{C_{\mathrm{m}}}\left(I-I_{\mathrm{K}}-I_{\mathrm{Na}}-I_{l}\right) \\
\dot{n}=\alpha_{n}(1-n)-\beta_{n} n \\
\dot{m}=\alpha_{m}(1-m)-\beta_{m} m \\
\dot{h}=\alpha_{h}(1-h)-\beta_{h} h,
\end{array}\right.
$$

where

$$
\begin{aligned}
I_{\mathrm{K}} & =\bar{g}_{\mathrm{K}} n^{4}\left(V-V_{\mathrm{K}}\right), \quad I_{\mathrm{Na}}=\bar{g}_{\mathrm{Na}} m^{3} h\left(V-V_{\mathrm{Na}}\right), \\
I_{l} & =\bar{g}_{l}\left(V-V_{l}\right) \\
\alpha_{n} & =\frac{0.01(V+10)}{e^{\frac{V+10}{10}}-1}, \quad \beta_{n}=0.125 e^{\frac{V}{80}} \\
\alpha_{m} & =\frac{0.1(V+25)}{e^{\frac{V+25}{10}}-1}, \quad \beta_{m}=4 e^{\frac{V}{18}} \\
\alpha_{h} & =0.07 e^{\frac{V}{20}}, \quad \beta_{h}=\left[e^{\frac{V+30}{10}}+1\right]^{-1},
\end{aligned}
$$

with the following parameter values: membrane capacitance $C_{\mathrm{m}}=1 \mu \mathrm{F} / \mathrm{cm}^{2}$; constant membrane potentials $V_{\mathrm{K}}=12$ $\mathrm{mV}, V_{\mathrm{Na}}=-115 \mathrm{mV}$, and $V_{l}=-10.6 \mathrm{mV}$; constants associated with membrane conductances $\bar{g}_{\mathrm{K}}=36 \mathrm{mS} / \mathrm{cm}^{2}$, $\bar{g}_{\mathrm{Na}}=120 \mathrm{mS} / \mathrm{cm}^{2}$, and the conductance $\bar{g}_{l}=0.3 \mathrm{mS} / \mathrm{cm}^{2} . I$ is the total current density through the membrane, and $I_{\mathrm{K}}, I_{\mathrm{Na}}$. and $I_{l}$ correspond to the current density due to potassium ions, sodium ions, and other ions, respectively. All current densities are given in $\mu \mathrm{A} / \mathrm{cm}^{2}$. Variables $n, m$, and $h$ are dimensionless variables corresponding to the proportion of the potassium inside the membrane, the proportion of activating molecules within the membrane, and the proportion of inactivating molecules outside it, respectively.

\section{B. The FitzHugh-Nagumo model}

The model obtained from a modified van der Pol's equation

$$
\left\{\begin{array}{l}
\dot{x}=c\left(y+x-x^{3} / 3+I\right) \\
\dot{y}=-(x-a+b y) / c,
\end{array}\right.
$$

is known as the FitzHugh-Nagumo model, ${ }^{9,34,39}$ and is considered to be a simplified version of the Hodgkin-Huxley model in the sense that it reproduces some of the main features of the dynamics. In model $(2),(a, b, c)$ are constant parameters and $I$ is a stimulus, that corresponds to membrane current in the Hodgkin-Huxley model. $x$ is usually identified with the membrane potential, and $y$ is the recovery variable. Model $(2)$ with $(a, b, c)=(0.7,0.8,3)$ was investigated in Ref. 9 with $I=-0.4$ and in Ref. 45 with $I(t)$ being a slowly varying stimulus within the range $-1.5 \leq I(t) \leq-0.4$.

\section{The Hindmarsh-Rose model}

The Hindmarsh-Rose equations are a three-equilibriumpoint model with adaptation ${ }^{17}$

$$
\left\{\begin{array}{l}
\dot{x}=y-a x^{3}+b x^{2}+I-z \\
\dot{y}=c-d x^{2}-y \\
\dot{z}=r\left[s\left(x-x_{1}\right)-z\right],
\end{array}\right.
$$


with $(a, b, c, d)=(1,3,1,5)$, where $x_{1}$ is a constant. The constant parameters $r$ and $s$ determine the dynamical response to a short pulse of depolarizing current. Here, $x$ is the membrane potential and $y$ is the recovery variable (as in the FitzHugh-Nagumo model). In this model, $z$ is an adaptation current that hyperpolarizes the cell. Similar to $y, z$ quantifies the transport of ions but now through slow channels.

The dynamics associated with $z$ is very slow compared to those of $x$ and $y$. The authors investigate three values for the current: $I=0.4, I=2$, and $I=4$. For $I=3.25$, a "random" (chaotic) burst structure has been reported.

\section{Izhikevich's spiking neuron Model}

The model

$$
\begin{gathered}
\left\{\begin{array}{l}
\dot{v}=0.04 v^{2}+5 v+140-u+I \\
\dot{u}=a(b v-u),
\end{array}\right. \\
\text { if } v \geq 30 \text { then } \mid \begin{array}{l}
v \leftarrow c \\
u \leftarrow u+d
\end{array}
\end{gathered}
$$

was proposed in Ref. 20 In Eq. (4), $\alpha \leftarrow \beta$ means that $\alpha$ receives the value of $\beta$. Model (4) combines aspects of the Hodgkin-Huxley model and of integrate-and-fire neuron models, and reproduces spiking and bursting behavior of known types of cortical neurons. In Eq. (4), $v$ represents the membrane potential and $u$, the membrane recovery variable; $(a, b, c, d)$ are constants and $I$ is the sum of the synaptic currents ( $I_{s y n}$, to be used in coupling neuron models) and injected dc currents $\left(I_{i n}\right)$. Depending on the parameter values, a rich variety of dynamical regimes are possible. For instance $(a, b, c, d)=(0.02,0.2,-50,2)$ with $I_{s y n}=10$ results in chattering ${ }^{20}$ and $(a, b, c, d)=(0.2,2,-56,-16)$ with $I_{s y n}=-99$ results in chaotic firing ${ }^{21}$ as evidenced by the first-return map to a Poincaré section (Fig. 1). The fact that a second-order model could produce chaos should not cause surprise because of the switching. In a sense, the state of the switch acts as a "hidden state variable." The Izhihevich's model is therefore a "truncated" model in the sense that all underlying mechanisms are not explicitly described.

\section{MATHEMATICAL AND NUMERICAL TOOLS}

In this section, we briefly review the observability coefficients and multivariate singular spectrum analysis (M-SSA).
Because of confusion in the literature as to the development of observability coefficients, ${ }^{43}$ here a brief historical overview is provided.

The concepts of observability and controllability for linear systems are due to Kalman. ${ }^{23}$ These were extended to nonlinear systems over a decade later as discussed. ${ }^{16}$ In both cases, the systems are classified either as observable or not. Bernard Friedland suggested computing a conditioning number of a symmetric matrix obtained from the linear observability or controllability matrices as a way of getting a continuous function of the parameters instead of a binary (either observable or not) classification. ${ }^{10}$ In fact, it was argued that although a similarity transformation of coordinates would not change the rank of the observability or controllability matrices, and therefore would not alter the resulting classification, the indices proposed by Friedland are sensitive to such a transformation, ${ }^{1}$ to changes in parameters and, in the nonlinear case, to the location in the state space. The concept of a continously varying quantification of observability was adapted to nonlinear dynamical systems, ${ }^{27,30}$ where the jacobian matrix of the vector field was used in the analysis. Later on, it was shown that the jacobian matrix of the map between the original and embedding spaces coincided with the nonlinear observability matrix based on Lie derivatives. ${ }^{29}$ Hence, the quantification of observability was then performed using such a matrix. The extension to multivariate embeddings and the relation to the Takens' theorem were presented in Ref. 2. The procedure proposed in Ref. 29 is briefly reviewed in Sec. III-A.

\section{A. Numerical observability coefficients}

Consider the autonomous system $\dot{\boldsymbol{x}}=f(\boldsymbol{x})$, where $\boldsymbol{x} \in$ $\mathbb{R}^{n}$ is the state vector and $f: \mathbb{R}^{n} \mapsto \mathbb{R}^{n}$ is the vector field. Consider further the measurement function $h: \mathbb{R}^{n} \mapsto \mathbb{R}$ such that $s(t)=h(\boldsymbol{x})$, where $s \in \mathbb{R}$ is referred to as the observable or recorded variable. The case for which $s \in \mathbb{R}^{p}, p>1$ has been investigated in Ref. 2 . The general observability matrix can be written as ${ }^{16}$

$$
\mathcal{O}_{s}(\boldsymbol{x})=\left[\begin{array}{ccc}
\frac{\partial \mathcal{L}_{f}^{0} h(\boldsymbol{x})}{\partial \boldsymbol{x}} & \ldots & \frac{\partial \mathcal{L}_{f}^{n-1} h(\boldsymbol{x})}{\partial \boldsymbol{x}}
\end{array}\right]^{\mathrm{T}},
$$
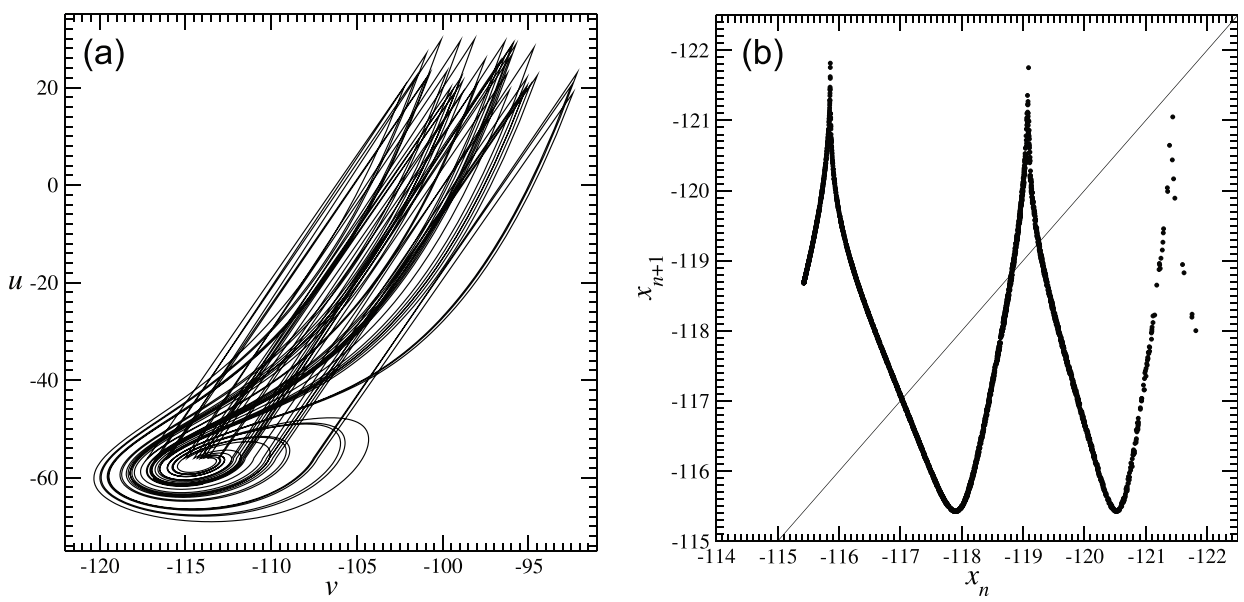

FIG. 1. Chaotic behavior produced by the Izhikevich's model. Parameter values: $(a, b, c, d)=(0.2,2,-56,-16)$ with $I_{\mathrm{syn}}=-99$. The six-branches first-return map to a Poincaré section of (4) is typical of a "funnel" chaotic behavior encountered in the Rössler system $^{38}$ for $a \approx 0.540, \quad b=2$ and $c=4$. (a) Chaotic attractor (b) Firstreturn map. 
where $\mathcal{L}_{f} h(\boldsymbol{x})$ is the Lie derivative of $h$ along the vector field $f^{19}$ and $s$ indicates that $\mathcal{O}_{s}(x)$ refers to the system observed from $s(t)$. In the case $h(\boldsymbol{x})$ returns, only one of the state variables matrix (5) can be rewritten as

$$
\mathcal{O}_{s}(\boldsymbol{x})=\left[\begin{array}{llll}
C & C \tilde{A} & \ldots & C \tilde{A}^{n-1}
\end{array}\right]^{\mathrm{T}},
$$

where $C=[10 \ldots 0]$ if $h(\boldsymbol{x})$ returns the first state variable, $C=\left[\begin{array}{llll}0 & 1 & 0 & \ldots\end{array}\right]$ if $h(\boldsymbol{x})$ returns the second, and so on. Also

$$
\tilde{A}^{j+1}=\left[\frac{\partial \mathcal{L}_{f}^{j} f_{i}(\boldsymbol{x})}{\partial \boldsymbol{x}}\right], \quad i=1,2, \ldots, n
$$

for $j=0, \ldots, n-2$, where

$$
\mathcal{L}_{f} f_{i}(\boldsymbol{x})=\frac{\partial f_{i}(\boldsymbol{x})}{\partial \boldsymbol{x}} \boldsymbol{f}(\boldsymbol{x})=\sum_{k=1}^{n} \frac{\partial f_{i}(\boldsymbol{x})}{\partial \boldsymbol{x}} f_{k}
$$

is the Lie derivative of the $i$ th component of the vector field $f$ and the higher-order derivatives can be recursively determined as

$$
\mathcal{L}_{f}^{j} f_{i}(\boldsymbol{x})=\mathcal{L}_{f}\left[\mathcal{L}_{f}^{j-1} f_{i}(\boldsymbol{x})\right],
$$

with $\mathcal{L}_{f}^{0} f_{i}(\boldsymbol{x})=f_{i}(\boldsymbol{x})$. If $\mathcal{O}_{s}(\boldsymbol{x})$ is singular then there is no global diffeomorphism between the original phase space and the $n$-dimensional space reconstructed using $s$ and $n-1$ successive derivatives of it. Because the system is nonlinear, often $\mathcal{O}_{s}(\boldsymbol{x})$ may become singular or nearly singular at specific regions of state space at which the original dynamics become poorly observable or nonobservable altogether. Hence, it is sometimes instructive to have an average measure of the numerical conditioning of $\mathcal{O}_{s}(\boldsymbol{x})$. In the present paper this is achieved averaging along a trajectory $\boldsymbol{x}(t)$ the quotient between the mininum and maximum eigenvalues of $\mathcal{O}_{s}(\boldsymbol{x})^{T} \mathcal{O}_{s}(\boldsymbol{x})$. The result is referred to as the observability coefficient $\delta_{s}$ for the system from the $s$ variable.

\section{B. Symbolic observability coefficients}

The advantage of the numerical observability coefficients is that they take into account the domain of the state space actually visited by the trajectory and, consequently, whether the neighorhood of the singular observability manifold is visited or not. Nevertheless, these observability coefficients are not normalized and cannot be used to compare different dynamical systems. To overcome such a problem, symbolic observability coefficients were introduced. ${ }^{28}$ The underlying idea is that the more complicated the determinant det $\mathcal{O}_{s}$ of the observability matrix, the less observable. Although the analytical computation of $\operatorname{det} \mathcal{O}_{s}$ can be a nearly impossible task for a five-dimensional rational system, the complexity of det $\mathcal{O}_{s}$ can be assessed simply by counting the number of linear, nonlinear and rational terms in it, without paying attention to its exact form. ${ }^{6}$ This is computed from the Jacobian matrix of the system which is transformed into the symbolic form, using $1, \overline{1}$, and $\overline{\overline{1}}$ for linear, nonlinear, and rational elements, respectively. The observability matrix is then constructed using symbolic algebra detailed in Ref. 6 . The symbolic observability coefficient is thus defined as

$$
\begin{aligned}
\eta_{s^{n}}= & \frac{N_{1}}{N_{1}+N_{\overline{1}}+N_{\overline{\overline{1}}}}+\frac{N_{\overline{1}}}{\left(\max \left(N_{1}, 1\right)+N_{\overline{1}}+N_{\overline{1}}\right)^{2}} \\
& +\frac{N_{\overline{\overline{1}}}}{\left(\max \left(N_{1}, 1\right)+N_{\overline{1}}+N_{\overline{\overline{1}}}\right)^{3}},
\end{aligned}
$$

where $N_{1}, N_{\overline{1}}$, and $N_{\overline{1}}$ are the numbers of symbolic terms 1 , $\overline{1}$, and $\overline{\overline{1}}$, respectively. These symbolic coefficients are very promising for assessing the observability of large systems and networks. ${ }^{31}$ The observability can be considered "good" when $\eta_{s^{3}}>0.75$, meaning that most likely the determinant of the observability matrix is linear if not constant; consequently the influence of the singular observability matrix is not very important. ${ }^{41}$

\section{Observability coefficients from data}

The procedures reviewed in Secs. III A and III B require the knowledge of the system equations. Motivated by the fact that in practice equations are not always available, an alternative procedure was proposed. ${ }^{3}$ However, observability is, by definition, related to the equations of the vector field. Hence, estimating coefficients from data is only an indirect way of assessing observability from some of its signatures found in a reconstructed (embedding) space, as explained next.

The rationale behind the method ${ }^{3}$ is that in the embedding space of a system with poor observability conveyed by a recorded time series, trajectories are either pleated or squeezed. $^{3}$ The SVDO (Singular Value Decomposition Observability) coefficients hence quantify, using the singular value decomposition (SVD) of a trajectory matrix, the local complexity of the reconstructed space. Simpler structures are associated with better observability whereas more complex local structures with poorer observability. A key point to be noticed here is that SVDO cannot quantify observability per $s e$, which by definition would require the vector field equations, but rather are indicators of the average local complexity of a reconstructed space, which often-but not always, as will be seen shortly-correlates with observability. In this paper we use time delay coordinates. Although the results will be reported for a given time delay and embedding dimension, numerical studies with different values of such parameters show no change in the ranking of the variables in terms of observability.

\section{Multivariate singular spectrum analysis}

Here, the use of the structured-varimax multivariate singular spectrum analysis (svM-SSA) for phase synchronization (PS) phenomena, originally proposed by Groth and Ghil, ${ }^{14}$ is briefly reviewed (for details, see Ref. 13). Recently, it was argued that the explanatory power of svMSSA on the mechanism of PS is greatly enhanced by the use of a single state variable, as compared to the original approach using all of them, as long as this variable provides 
good observability of the dynamics as compared to the rest. $^{35}$

Consider $J$ coupled oscillators and the respective time series of length $N$ of the variable that provides the best observability. Each time series is split in windows of $m$-data points, and a full augmented "trajectory matrix" $\mathbf{X}=$ $\left[\mathbf{X}_{1}, \ldots, \mathbf{X}_{J}\right] \in \mathbb{R}^{N-m+1, J m}$ is formed by concatenating the individual time series. In the M-SSA literature, the parameter $m$ is called the window width or embedding dimension: we prefer to use the former term. The svM-SSA starts by the eigendecomposition of the lag-covariance matrix $\mathbf{C}=$ $\mathbf{X}^{T} \mathbf{X} /(N-m+1)$ as $\boldsymbol{\Lambda}=\mathbf{E}^{T} \mathbf{C E}$. To overcome a known mixture of the eigenvectors related to the individual subsystems, a structured-varimax rotation ${ }^{14,36}$ is performed on the first $S$ eigenvectors, $\mathbf{E}_{S}^{*}=\mathbf{E}_{\mathbf{S}} \mathbf{T}$. Finally, the modified variances $\left\{\lambda_{k}^{*}\right\}_{k=1}^{S} \equiv \operatorname{diag}\left(\boldsymbol{\Lambda}_{S}^{*}\right)$ are obtained through $\boldsymbol{\Lambda}_{S}^{*}=\mathbf{T}^{T} \boldsymbol{\Lambda}_{S} \mathbf{T}$ and they encode information about the underlying structure of the data: a single high value is related to a trend; pairs of nearly equal values reflect the oscillatory modes; near zero values are associated with noncoherent oscillations and will be referred to as the noise floor. Hence, it is possible to infer about PS, without any prior definition of how to estimate the oscillators phases, from the evolution of $\lambda_{k}^{*}$ pairs associated to the oscillatory modes in the data, as schematically illustrated in Fig. 2 for $J=4$ idealized oscillators.

\section{RESULTS ON OBSERVABILITY}

Here we provide numerical results about the observability of the models in Sec. II which were integrated using a 4th-order Runge-Kutta algorithm with integration step $h=0.01$.
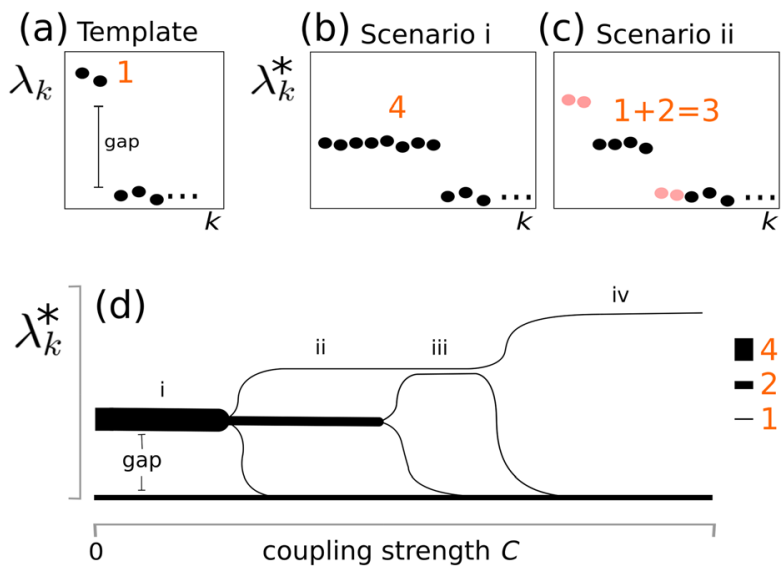

FIG. 2. Schematic representation of an svM-SSA for $J=4$ idealized coupled oscillators. (a) The template shows the signature of a single oscillatory mode, identified by a unique $(\mu=1) \lambda_{k}$ pair separated from the "noise floor" by a clear gap. (b) Prior PS, four distinct oscillatory modes are identified $(\mu=4)$. (c) The onset of the first PS cluster is identified by one $\lambda_{k}^{*}$ pair becoming larger as another pair simultaneously merges with the noise floor (both pairs are indicated in orange). (d) $\lambda_{k}^{*}$ for an increasing coupling strength $C$ : (i) oscillators start non-synchronized-like in (b); (ii) two oscillators form a PS cluster when a pair of $\lambda_{k}^{*}$ increases and another pair falls to the noise floor - as the orange pairs in (c); (iii) other two oscillators form a second PS cluster, and again there is an increase in a $\lambda_{k}^{*}$ pair while the other merges with the noise floor (and $\mu=2$ ); (iv) finally, the two clusters merge into a single PS one. The number $\mu$ of $\lambda_{k}^{*}$ pairs above the noise floor (equivalently, of different oscillatory modes detected) is indicated by the thickness of the lines.

\section{A. The Hodgkin-Huxley model}

Given the nonlinearity and dimension of the Hodgkin-Huxley model and due to the recursive computation of Lie derivatives, the observability matrices are too large (hundreds of entries obtained by symbolic computation) to be shown here. Also, since (1) is a biophysicallybased model, when investigating observability properties one should keep in mind what variables are actually recordable. Out of the four state variables of this model only the membrane potential $V$ is recordable, the other variables being dimensionless quantities. Nonetheless, for the sake of completion, we here report the observability coefficients for the four state variables in Table I. The symbolic Jacobian matrix of the Hodgkin-Huxley model is

$$
\mathcal{J}^{\text {sym }}=\left[\begin{array}{cccc}
\overline{1} & \overline{1} & \overline{1} & \overline{1} \\
\overline{\overline{1}} & \overline{1} & 0 & 0 \\
\overline{\overline{1}} & 0 & \overline{1} & 0 \\
\overline{\overline{1}} & 0 & 0 & \overline{1}
\end{array}\right]
$$

from which the symbolic observability coefficients shown in Table I can be obtained.

Because the state variables $n, m$, and $h$ cannot be measured, a different procedure was followed that is made available by using the indirect assessment of observability from data proposed in Ref. 3. Hence the membrane potential $V$ and the currents $I_{\mathrm{K}}, I_{\mathrm{Na}}$, and $I_{l}$ were considered as candidate variables to be used in reconstructing a phase space for the dynamics. The SVDO coefficients shown in Table I were computed for $I=-10$ using a 5-dimensional embedding

TABLE I. Observability coefficients for the different models investigated in this work. (*) for $I=-1$.

\begin{tabular}{|c|c|c|c|}
\hline \multicolumn{4}{|c|}{ Hodgkin-Huxley model } \\
\hline & $\delta_{\mathrm{s}}$ & $\eta_{s^{4}}$ & $S_{\mathrm{s}}$ \\
\hline$V$ & $1.11 \times 10^{-7}$ & 0.12 & $0.11 \pm 0.001$ \\
\hline$n$ & $1.09 \times 10^{-6}$ & 0.19 & $0.09 \pm 0.002\left(I_{\mathrm{K})}\right.$ \\
\hline$m$ & $5.14 \times 10^{-9}$ & 0.19 & $0.05 \pm 0.002\left(I_{\mathrm{Na})}\right.$ \\
\hline$h$ & $4.90 \times 10^{-6}$ & 0.19 & $0.17 \pm 0.003\left(I_{1}\right)$ \\
\hline & \multicolumn{3}{|c|}{ FitzHugh-Nagumo model } \\
\hline & $\delta_{\mathrm{s}}$ & $\eta_{s^{2}}$ & $S_{\mathrm{s}}$ \\
\hline$x$ & $0.04^{(*)}$ & 1.00 & $0.30 \pm 0.02$ \\
\hline \multirow[t]{3}{*}{$y$} & 0.09 & 1.00 & $0.21 \pm 0.01$ \\
\hline & \multicolumn{3}{|c|}{ Hindmarsh-Rose model } \\
\hline & $\delta_{\mathrm{s}}$ & $\eta_{s^{3}}$ & $S_{\mathrm{s}}$ \\
\hline$x$ & $6.32 \times 10^{-4}$ & 0.25 & $0.67 \pm 0.16$ \\
\hline$y$ & $3.31 \times 10^{-4}$ & 0.56 & $0.45 \pm 0.16$ \\
\hline \multirow[t]{3}{*}{$z$} & $2.90 \times 10^{-6}$ & 1.00 & $186.63 \pm 33.68$ \\
\hline & \multicolumn{3}{|c|}{ Izhikevich's model } \\
\hline & & $\eta_{s^{2}}$ & $S_{\mathrm{s}}$ \\
\hline$u$ & & 1.00 & $0.75 \pm 0.29$ \\
\hline$v$ & & 1.00 & $8.38 \pm 0.51$ \\
\hline
\end{tabular}


space and a common delay time ( $\tau=100$ sampling intervals) for the three variables. The reported values are mean plusminus one standard deviation over ten Monte Carlo runs where the initial conditions were taken randomly from Gaussian distributions: $\mathcal{N}(-60,1)$ for $V(0), \mathcal{N}\left(0.3,0.01^{2}\right)$ for $n(0), \mathcal{N}\left(0,0.01^{2}\right)$ for $m(0)$ and $\mathcal{N}\left(0.55,0.01^{2}\right)$ for $h(0)$. The results show that the best measured signals for reconstructing a phase space using delay coordinates are $V$ and $I_{l}$. The simple (afine) relationship between such signals suggests that they are somewhat equivalent in what concerns observability.

\section{B. The FitzHugh-Nagumo model}

The observability matrix for model (2) when $x$ is recorded is

$$
\mathcal{O}_{x}=\left[\begin{array}{cc}
1 & 0 \\
-c\left(x^{2}-1\right) & c
\end{array}\right],
$$

with determinant $\operatorname{det}\left(\mathcal{O}_{x}\right)=c$, hence unless $c=0$ the system is observable from the $x$ variable, although observability could be poor for very small values of $c$. Notice that for $0<$ $c \ll 1$ the model (2) becomes a singular perturbed system. Recording $y$ yields the observability matrix

$$
\mathcal{O}_{y}=\left[\begin{array}{cc}
0 & 1 \\
-1 / c & -b / c
\end{array}\right],
$$

also with constant determinant $\operatorname{det}\left(\mathcal{O}_{y}\right)=1 / c$. Hence for very high values of $c$, the $y$ variable conveys worse observability of the dynamics.

$\mathcal{O}_{x}$, that depends on $x$, varies along the limit cycle which, in turn is affected by $I$. Contrary to this $\mathcal{O}_{y}$ is constant throughout the phase space and is not influenced by the stimulus. Both observability coefficients $\delta_{x}$ and $\delta_{y}$ are of the same order of magnitude for the chosen parameters. Hence, unless $c$ is very large or very small, both state variables are comparable in what concerns observability, although measuring $y$ ensures more uniform performance along the limit cycle. Similar conclusions were attained (Table I) using the symbolic observability matrix

$$
\mathcal{O}_{x}^{\text {sym }}=\left[\begin{array}{ll}
1 & 0 \\
1 & 1
\end{array}\right]
$$

for which Det $\mathcal{O}_{x}^{\text {sym }}=1 \otimes 1$ where $\otimes$ is the multiplicative law between the symbols as defined in Ref. 6 .

The SVDO coefficients for the Fitzhugh-Nagumo model, shown in Table I, confirm that the observability of both variables are comparable and that the embedding space reconstructed with $y$ is rather more homogeneous. These values were computed for $I=-0.4$ using a 3-dimensional embedding space and a common delay time $(\tau=100$ sampling intervals) for the three variables. In the ten Monte Carlo runs, the initial conditions of both state variables were taken from $\mathcal{N}(0,1)$.

\section{The Hindmarsh-Rose model}

The observability of the Hindmarsh-Rose (HR) model has been considered recently. In Ref. 35, a modified version of (3) was considered with linearly transformed coupled equations, ${ }^{5}$ and in Ref. 41 symbolic observability coefficients $^{28}$ were computed. So here we compute the observability coefficients as used in Ref. 29 for model (3). Following Ref. 41, we use $(a, b, c, d)=(1,3,1,5)$ and $\left(r, s, x_{1}, I\right)$ $=\left(0.001,4,-\frac{1+\sqrt{5}}{2}, 3.318\right)$. The observability matrices for model (3) are

$$
\mathcal{O}_{x}=\left[\begin{array}{ccc}
1 & 0 & 0 \\
2 b x-3 a x^{2} & 1 & -1 \\
O_{31}^{x} & O_{32} & 3 a x^{2}-2 b x+r
\end{array}\right]
$$

where $O_{31}^{x}=\left(2 b x-3 a x^{2}\right)^{2}-r s-2 d x+(2 b-6 a x)\left(-a x^{3}\right.$ $\left.+b x^{2}+I+y-z\right)$ and $O_{32}^{x}=-3 a x^{2}+2 b x-1 . \quad \mathcal{O}_{x}$ becomes singular for $r=1$ because in that case the two last columns become linearly dependent, in fact, det $\left(\mathcal{O}_{x}\right)=r$ -1 . Singularity is not expected to happen for the usual values of $r$, although a negative value for the determinant may indicate that the original and reconstructed spaces are topologically equivalent, but not orbitally equivalent, that is the direction of the flow may be different in each space. As discussed in Ref. 33, this negative determinant could suggest that a higher-dimensional space should be used to recover full observability. The observability matrix from the $y$ variable is:

$$
\mathcal{O}_{y}=\left[\begin{array}{ccc}
0 & 1 & 0 \\
-2 d x & -1 & 0 \\
O_{31}^{y} & 1-2 d x & 2 d x
\end{array}\right]
$$

where $O_{31}^{y}=-2 d\left(-x-a x^{3}+b x^{2}+I+y-z\right)-2 d x(2 b x$ $\left.-3 a x^{2}\right)$. $\mathcal{O}_{y}$ becomes singular at $x=0$, in fact det $\left(\mathcal{O}_{y}\right)=4 d^{2} x^{2}=100 x^{2}$, for the parameters used. Although this situation could happen several times during firing, the associated dynamics are so fast that the time the system spends close to $x=0$ is so short that this does not pose practical observability problems. $^{11}$ Finally, the observability matrix when $z$ is recorded is

$$
\mathcal{O}_{z}=\left[\begin{array}{ccc}
0 & 0 & 1 \\
r s & 0 & -r \\
r s\left(2 b x-3 a x^{2}\right)-r^{2} s & r s & r(r-s)
\end{array}\right],
$$

which becomes singular at $r s=0$. In this case $\rho\left[\mathcal{O}_{z}\right]=1$, where $\rho$ stands for the rank. This, added to the fact that $r$ is already quite small, shows that $z$ conveys poor observability of the system. It is interesting to notice that although det $\left(\mathcal{O}_{z}\right)=r^{2} s^{2}=1.6 \cdot 10^{-5}$ is constant this does not imply good observability.

In order to quantify observability, coefficients were computed for $(a, b, c, d)=(1,3,1,5),\left(r, s, x_{1}\right)=(0.001,4$, $-\frac{1+\sqrt{5}}{2}$ ) and $I=3.318$ (Table I). The fast variables provide better observability than the slow one $z$. 
The symbolic observability matrix reads

$$
\mathcal{O}_{x}^{\text {sym }}=\left[\begin{array}{ccc}
1 & 0 & 0 \\
\overline{1} & 1 & 1 \\
\overline{1} & \overline{1} & \overline{1}
\end{array}\right]
$$

and $\eta_{x^{3}}=0.25$. Nevertheless, $\operatorname{det} \mathcal{O}_{x}=r-1$ and is clearly not dependent on the location in the state space. It is never singular unless $r \neq 1$. A global diffeomorphism could be therefore expected, that is, a full observability should be provided by this variable although it could be very illconditioned, and therefore poor. This is one of the rare cases where two nonlinear terms in the computation of the determinant cancel each other; the symbolic computation is therefore very different from an analytical computation. Note that as the dimension of the system increases, this situation becomes less likely. It has been argued that from the symbolic point of view, the coefficient $\eta_{x^{3}}$ should be corrected to be equal to $1 .{ }^{41}$ As long as $r$ is significantly different from 1 , taking $\eta_{x^{3}}=1$ should be a fair estimation of the observability. From the two other symbolic observability matrices, we obtained $\eta_{y^{3}}=0.56$ and $\eta_{z^{3}}=1$.

The symbolic observability coefficient $\eta_{x^{3}}$-if not corrected-would suggest a rather poor observability of the dynamics underlying the Hindmarsh-Rose system from variable $x$. In fact, and contrary to what is provided from the determinant point of view, variable $x$ does not provide a good observability of the underlying dynamics, when parameter $r$ has a small value, e.g., $r<0.01$. This is confirmed by a differential embedding induced by variable $x$ of the attractor produced by the Hindmarsh-Rose system [Fig. 3(a)] which clearly shows that the chaotic nature of the behavior is poorly evidenced, contrary to what is observed when the differential embedding induced by variable $z$ is used [Fig. 3(c)]. For very small r-values, e.g., $r<0.01$, the symbolic observability coefficient $\eta_{x^{3}}$ does not properly reflect the observability. This is one of the cases where the dynamics is directly responsible for particular difficulties in distinguishing the different states of the system.

The SVDO coefficients for $I=3.318$ are shown in Table I and for $I=2$ they are $S_{x}=0.42 \pm 0.04, S_{y}=0.20 \pm 0.03$, and $S_{z}=19.45 \pm 0.61$. These values were computed over ten Monte Carlo runs where the initial conditions of the three state variables were taken from $\mathcal{N}\left(0,0.1^{2}\right)$; using a 4 th dimensional embedding space and a common delay time $(\tau$ $=100$ sampling intervals) for the three variables. Numerical experimentation with other values did not change the ranking of the variables.

For both values of $I$ we find that $x$ and $y$ variables have similar features (as for $\delta_{x}$ and $\delta_{y}$ ), the difference being that $S_{z}$ suggests that $z$ provides better "observability." This was also the case of the symbolic observability coefficients. The chaotic nature of the reconstructed attractors - using delay or derivative coordinates-is better evidenced by variable $z$. The values of the observability coefficient $S_{s}$ also confirm these results. This does not necessarily mean that $z$ is a good observable. In fact, the fast dynamics (spikes) are practically invisible from $z$-which corresponds to the flat bottom of the
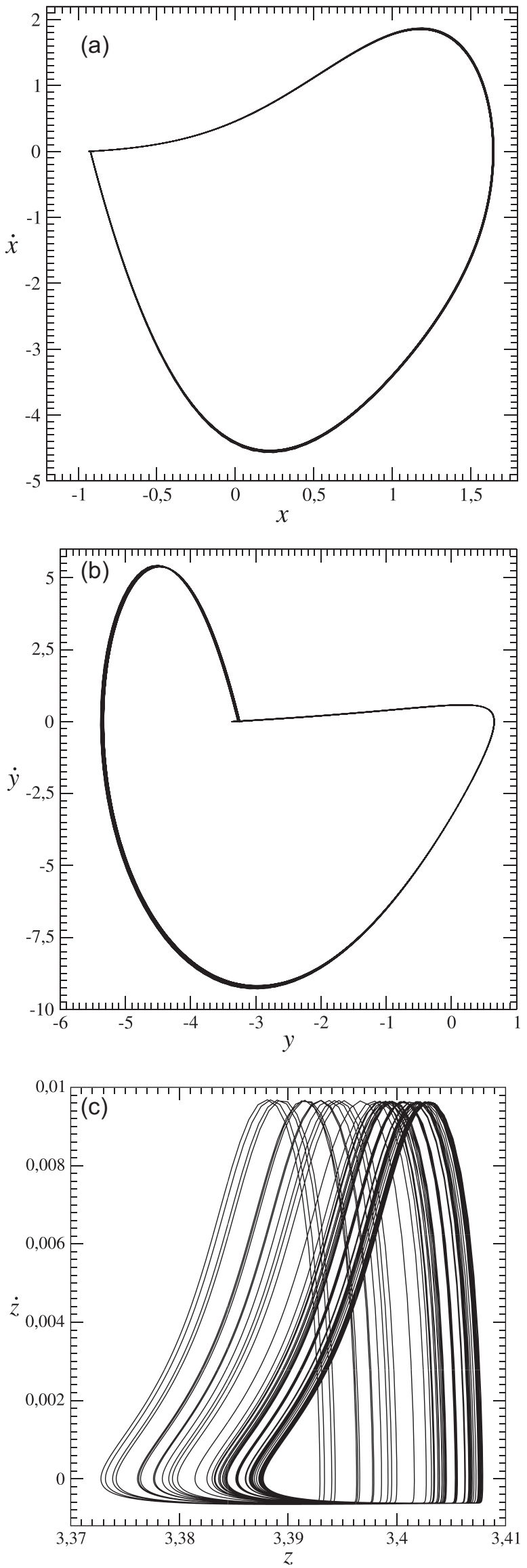

FIG. 3. Plane projection of the differential embedding induced by each variable of the Hindmarsh-Rose system. Top left: $x$ - $\dot{x}$ plane, top right: $y-\dot{y}$ plane, and bottom: $z-\dot{z}$ plane. 
attractor in Fig. 3(c). It seems that $z$ is the best observable for slow dyamics (chaos) whereas $x$ and $y$ convey information on the spikes (chattering).

\section{The Izhikevich's spiking neuron model}

The observability for model (4) poses interesting challenges because of the switching mechanism which is not produced by the differential equations. Also, the discontinuity induced by the switching poses theoretical difficulties to the application and interpretation of Lie derivatives. The computation of observability coefficients for this system is left for future research.

Because the symbolic observability coefficients do not depend on the parameters, the same values $\eta_{u^{2}}=\eta_{v^{2}}=1$ were obtained for both chattering and chaotic regimes. These results suggest that any of these two variables offer a full observability of the state variables $u$ and $v$, but not of the switching. It must be clear that by construction this is an approximation since the switching mechanism is not fully described in terms of the differential equation (at least a third variable would be necessary for this) and, consequently, there is no available technique so far to rigorously assess the observability of such a system.

The SVDO coeficients $\left(d_{\mathrm{e}}=3\right.$ and $\tau=100$ sampling intervals) for model (4) in the chaotic regime are shown in Table I. For chattering dynamics, they are $S_{v}=0.86 \pm 0.27$, $S_{u}=0.17 \pm 0.12$. In both regimes, the initial conditions were chosen randomly over ten Monte Carlo simulations from the Gaussian distributions: $\mathcal{N}\left(-65,10^{2}\right)$ for $v(0)$ and $\mathcal{N}\left(-130,10^{2}\right)$ for $u(0)$. It should be pointed out that the discontinuities of the trajectories in the state space produced by model (4) might have some unknown effect on the computation of the SVDO coefficients. It is the first time that such coefficients are computed from discontinuous data.

The results concerning observability are summarized in Table I. It seems that observing the dynamics underlying neurons is rather challenging because getting a reconstructed state portrait with a good observability of the original state space is not an easy task: this is not only due to the way the mechanisms are described in terms of equations but also due to the different time scales often encountered in neuron dynamics.

\section{SYNCHRONIZATION IN NEURON NETWORKS}

In this section, we provide numerical evidence that PS in networks of neuron models can be detected without estimating the phase, by using the svM-SSA with a single variable approach (see Sec. III D).

This is illustrated by two examples with different aims. The first example aims at illustrating the main aspects of the svM-SSA in a simple problem. The second example explores the feasibility of using the svM-SSA in the synchronization analysis of coupled neurons in a large network. Before discussing the examples, however, an important aspect must be addressed.

Let $\dot{\boldsymbol{x}}_{j}=f_{j}\left(\boldsymbol{x}_{j}, \boldsymbol{v}_{j}\right), j=1, \ldots, J$ be a network of $J$ coupled neuron models, where $\boldsymbol{x}_{j} \in \mathbb{R}^{n}$ is the state vector of the $j$ th model and $\boldsymbol{v}_{j}$ is a vector of coupling variables from other neurons. Also, let $h: \mathbb{R}^{n} \rightarrow \mathbb{R}$ be a measuring function such that $h\left(\boldsymbol{x}_{j}\right)=s_{j}(t)$. From the set of time series $s_{j}(t), j=1, \ldots, J$, it is desired to detect phase synchronization using svM-SSA.

In this framework there are two types of variables, and it is vital to see the difference between them. On the one hand, the variables in $v$ are used to couple the neuron models. Many ways of achieving this have been reviewed in Ref. 12 . It has been recently pointed out that neither controllability nor observability are generally useful in choosing the variables for coupling. ${ }^{4}$ On the other hand, there is the variable $s_{j}(t)$ that is used for analysis, i.e., the variable used to detect phase synchronization using svM-SSA. It has been argued that in choosing $s_{j}(t)$ the use of observability is most important. ${ }^{35}$ Hence, the results are relevant in practice due to the fact that the variable experimentally measured provides good observability of the system dynamics (Sec. III D).

\section{A. The FitzHugh-Nagumo model}

Consider a chain of $J=5$ diffusively coupled FitzHughNagumo neuron models

$$
\left\{\begin{array}{l}
\dot{x}_{j}=c\left(y+x-\frac{x^{3}}{3}+z\right)+C\left(x_{j+1}-2 x_{j}+x_{j-1}\right) \\
\dot{y}_{j}=-(x-a+b y) / c
\end{array}\right.
$$

with $j=1, \ldots, J$ with free boundary conditions $x_{0}=x_{1}$ and $x_{J+1}=x_{J}$, where $C$ is the coupling strength. Along this section, data was generated by integrating (19) with integration step $h=0.01$ time units (t.u.) for a total time $t_{\text {sim }}=450$ t.u., using a 4th-order Runge-Kutta algorithm. The first $t_{\text {trans }}=50$ t.u. were discarded, and the time series of $x$ and $y$ were sampled with $t_{\mathrm{s}}=0.7$ t.u. Also $(a, b)=(0.7,0.8)$ and detuning $c_{j}=c_{1}+(j-1) \Delta c$, with $c_{1}=3, \Delta c=0.2$ and $I=-4$. An interesting study on the observability and controllability of three-node networks with Fitzhugh-Nagumo models at each node has been discussed in Ref. 44.

Before starting the synchronization analysis, one needs to know the specific template of the oscillatory dynamics of one oscillator. Figure 4 shows the power spectrum of the $x$ time series and the 20 leading svM-SSA singular values for a single neuron model. The svM-SSA window width was $m=31\left(\approx 2 T / t_{\mathrm{s}}\right)$, covering almost two oscillatory periods. The template in Fig. 4(b) shows that a single oscillator will be identified as two leading pairs of singular values $\left(\lambda_{1,2}\right.$ and (a)

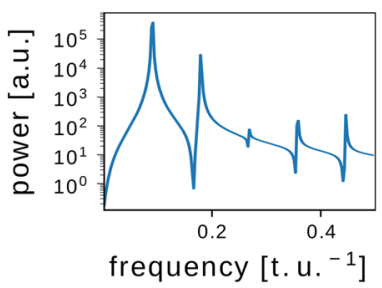

(b)

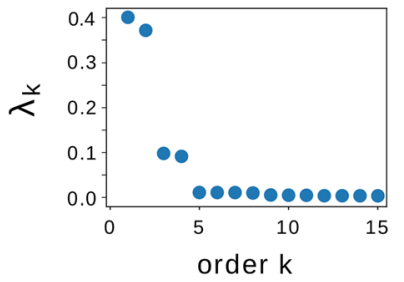

FIG. 4. (a) Power spectrum density with the fundamental frequency at $\approx 0.09 \mathrm{~Hz}$ and (b) svM-SSA template analysis of a FitzHugh-Nagumo neuron. Two oscillatory modes are predominant, a stronger $\left(\lambda_{1,2}\right)$ and a weaker one $\left(\lambda_{3,4}\right)$. 
$\lambda_{3,4}$ ), which are associated with two dominant modes seen in Fig. 4(a).

We illustrate the use of the svM-SSA by investigating the synchronization dynamics of the chain for an increasing coupling strength $C \in[0,0.06]$. A crosscheck of the PS results was done by: (a) computing the mean frequencies, $\Omega_{j}$, as the slope of a linear least squares fit of the instantaneous phase $\phi_{j}(t)$, which, in turn, was estimated through the analytic signal based on the Hilbert transform. Evidence for PS was verified through two criteria: (i) the mean frequency locking $\Omega_{j}=\Omega_{i}$ (weak PS condition); (ii) the phase entrainment $\Delta \phi_{i, j}(t)=\left|\phi_{i}(t)-\phi_{j}(t)\right|<$ const (strong PS condition). For the sake of completeness, also a visual inspection of the spatiotemporal patterns in $x_{j}(t)$ is provided, as it is usual in neuroscience.

Figure 5 shows the results. The following features are worth noticing. First, at low values of the coupling strength (e.g., at $C=C_{1}$, first dashed vertical line) the five oscillators have different frequencies $\Omega_{j}$, suggesting no PS. Before $C=C_{2}$, the onset of PS is detected by the increasing value of a $\lambda^{*}$ pair with a simultaneous drop of other pair to the noise floor [Fig. 5(b)]. Only later, at $C=C_{2}$ PS is suggested by the condition $\Omega_{2}=\Omega_{3}$. At $C=C_{3}$ there are two PS clusters indicated by $\lambda_{1,2}^{*}$ and $\lambda_{3,4}^{*}$ and confirmed by two different average

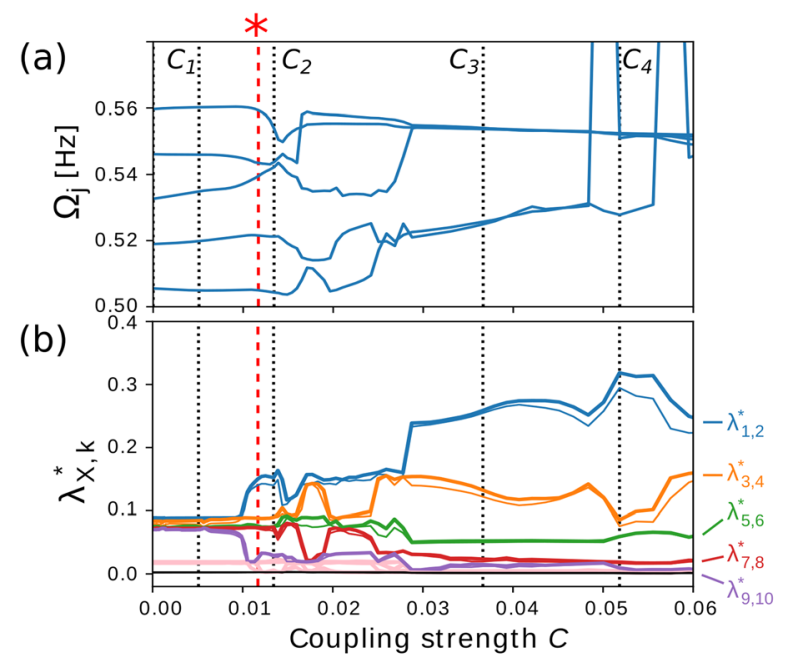

(c)
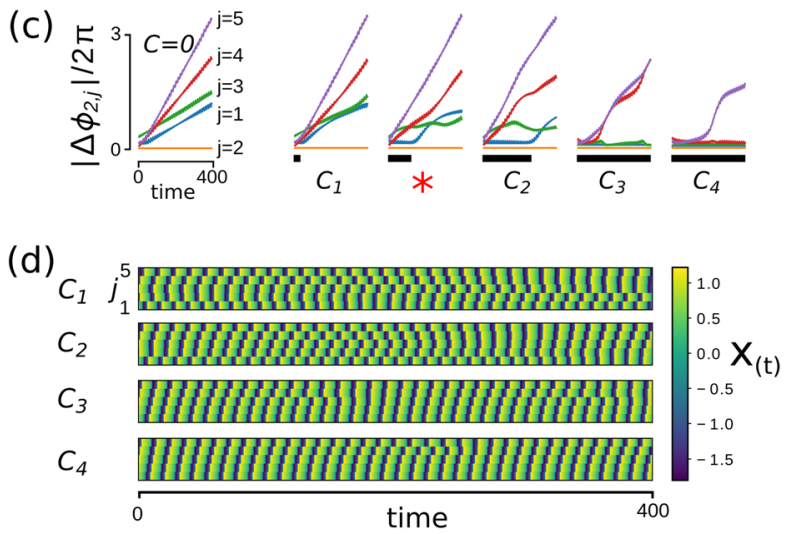

FIG. 5. Results for $J=5$ detuned FitzHugh-Nagumo neurons. (a) Mean observed frequencies: $\Omega_{1}$ (top) to $\Omega_{5}$ (bottom), (b) eigenvalues obtained by svM-SSA show the PS clustering (see text) (c) instantaneous phase difference $\left|\Delta \phi_{2, j}\right| / 2 \pi$, and (d) $x_{j}(t)$ spatiotemporal patterns. frequencies. The pair $\lambda_{1,2}^{*}$ is related to the main oscillatory mode of one cluster whereas $\lambda_{3,4}^{*}$ is related to the other one. At $C=C_{4}$, it is possible to devise a "jump" in the mean frequency associated with poor or inappropriate frequency estimation (e.g., due to phase slips). Such jumps were obtained regardless of how the phase was estimated (i.e.,, $\arctan (y / x)$ or through a Poincaré section, not shown). Since the svMSSA does not require the computation of phases, no "jumps" are present. This robustness represents another advantage of the method.

The phase entrainment condition $\Delta \phi_{2, j}(t)<$ const [Fig. 5 (c)], with oscillator $j=2$ taken as the reference, corroborates the results obtained with the svM-SSA. Oscillators $j=1,2$ are phase locked $\Delta \phi_{2,1}(t) \approx 0$ at $C<C_{2}$ (red star) for almost one third of the total observed time (thick black strip just below the curve $\left.\Delta \phi_{2,2}(t)=0\right)$. Also, $\Delta \phi_{2,3}(t)$ seems to be bounded as well, showing that PS onset is, in fact, due the cluster formed by oscillators $j=1,2$ and 3 indicated by the increase of $\lambda_{1,2}^{*}$. At $C=C_{4}$ oscillator $j=5$ suffers phase slips, which is associated with the aforementioned jump in the mean frequency plot.

Finally, parallel lines in the spatiotemporal patterns shown in Fig. 5(d) indicate PS. So, clearly, the scenario for $C=C_{4}$ is visually more synchronized than, say, for $C=C_{2}$. Also, a line broken into two parts (see $C=C_{3}$ ) is evidence for two PS clusters. Although such information could be extracted from the spatiotemporal patterns, the use of these plots is more subjective than the svM-SSA.

\section{B. The Izhikevich's spiking neuron model}

In the previous example, the svM-SSA was used in the context of a few oscillators. But computational investigations on neuronal dynamics also include networks with a massive number of neurons such as $J \sim 10^{3}$ up to $10^{6}$ and above. For example, a network model of the mammalian thalamocortical system exhibited polychronous activity only with $J>10^{4}$ neurons. $^{22}$

Hence, in this section a numerical experiment using a network with $J=1000$ excitatory Izhikevich neuron models is reported. Such a model has both biological plausibility and computational efficiency, and is able to emulate nearly 20 neuro-computational properties of biological spiking neurons. ${ }^{21}$

In model (4), each neuron receives a total current $I$, which is computed as

$$
I^{j}=I_{\text {in }}^{j}+g I_{\text {syn }}^{j}, \quad \text { where } I_{\text {syn }}^{j}=\sum_{i=1}^{J} S(j, i),
$$

and the synaptic weight matrix $S \in \mathbb{R}^{J \times J}$ quantifies how neurons $i=1, \ldots, J, i \neq j$ interact with neuron $j$. $S$ has $J=N_{\mathrm{e}}$ $+N_{\mathrm{i}}$ columns that correspond to the number of excitatory and inhibitory neurons. Here a population of excitatory neurons is considered, and then $N_{\mathrm{e}}=1000$ and $N_{\mathrm{i}}=0$. The entries of $S$ were selected from the uniform distribution $\mathcal{U}[0,0.5]$. In this example, following Refs. 21 and 24 $\left(a_{j}, b_{j}, c_{j}, d_{j}\right)=(0.02,0.2,-65,8)+(0,0,15,-6) r_{j}^{2}$, where $r_{j} \sim \mathcal{U}[0,1]$. The Euler integration method was used with $\left(h, t_{\mathrm{s}}, t_{\text {sim }}, t_{\text {trans }}\right)=(0.5,1,1000,0) \mathrm{ms}$. Fifty equally space 
(a)

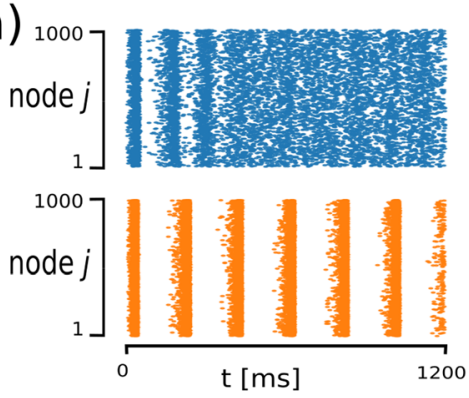

(c)

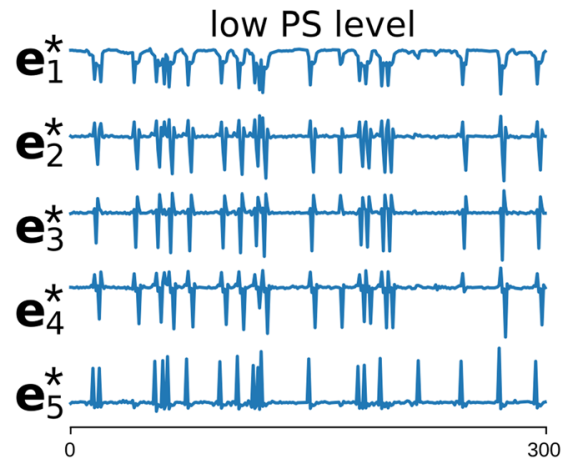

(b)

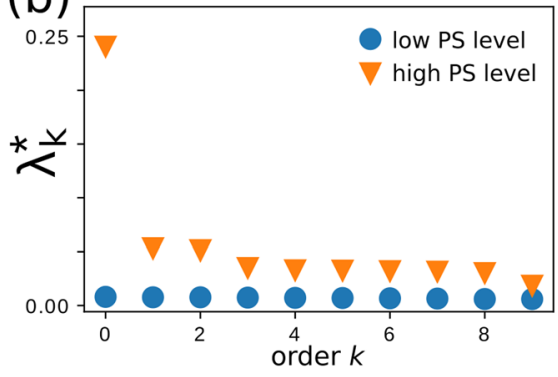

high PS level

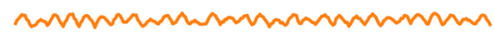
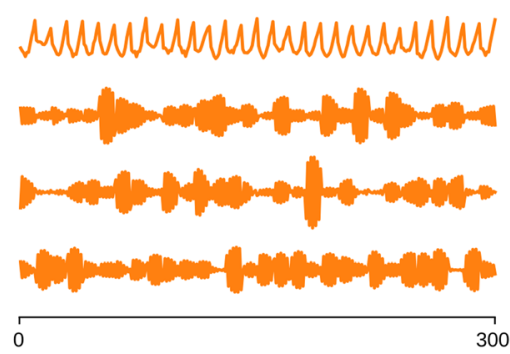

FIG. 6. Signatures of the svM-SSA for the $N=1000$ pulse-coupled Izhikevich neuron models. (a) Raster plots show two levels of synchronism, corresponding to low PS ( $g=0.4$, top panel) and high PS ( $g=0.5$, bottom panel). (b) Associated $\lambda_{k}^{*}$ spectra and (c) the five leading (respective) eigenvectors. values of $g \in[0.4,0.5]$ were taken. The same randomly generated synaptic weight matrix was used in all simulations.

The relevant dynamical information is coded in the occurrences of spikes and not in their magnitude. In view of this, a so-called spike raster time series $x_{j}(t)$ is produced from $v_{j}(t)$. Such a series is formed of 0 and $1 \mathrm{~s}$. Whenever a neuron fires $\left(v_{j}(t) \geq 30\right) x_{j}(t)=1$, which is otherwise 0 . Figure 6(a) shows the raster spike plots for $g=\{0.4,0.5\}$, which show low and high synchrony levels, respectively.

Due to the large number of neurons in this simulation, what is of interest is the collective dynamics that results from the global correlation presented by the 1000 neurons when they fire. If firing is totally uncorrelated, no global pattern is recognizable. This is the case at the end of the top panel shown in Fig. 6(a). When there is correlation of individual firing patterns, then collective evidence is recognizable as stripes in the raster plots as in the bottom panel in Fig. 6(a). The greater the correlation, the more well defined such stripes appear. The individual firing pattern can be represented by the sequence $[0,1,0]$, which has a duration of $3 \mathrm{~ms}$ in the $x$ time series. The window length should be such as to enable the lagcovariance matrix $\mathbf{C}$ (see Sec. III D) to capture correlations at such time scales. In this example $m=10 \mathrm{~ms}$ was chosen, but values as small as $m=4 \mathrm{~ms}$ yielded similar results.

There is also a slow time scale $(\approx 200 \mathrm{~ms})$ which is related to how the stripes in the raster plots-due to the collective dynamics - propagate through time, e.g., rapidly decaying as in the top panel in Fig. 6(a), or slowly decaying as in the bottom panel. The analysis of such a time scale using svM-SSA would require $m \approx 400$ and would result in a $\mathbf{C} \in \mathbb{R}^{J m \times J m}$ with a $J m=4 \times 10^{5}$ lag-covariance matrix. Such analysis is not viable at this stage.

The ten leading eigenvalues $\lambda_{k}^{*}$ and respective five eigenvectors $\mathbf{e}_{k}^{*}$ are shown in Figs. 6(b) and 6(c). The structured varimax rotation was performed with 20 eigenvectors to prevent over-rotation (but values as large as 2000 provided similar results). Note that a vector $\mathbf{e}_{k}^{*}$ is formed by $J$ segments of length $m$, each one associated with an oscillator $j^{14,35}$ (only the first 30 segments are shown in the plots). They provide a collection of features that forms the "skeleton" of the dynamical behavior structure. ${ }^{7,15}$ In the left panel of Fig. 6(c), no similar pattern of behavior was detected in the time series $x_{j}$ for the low synchrony level. Hence, all the eigenvectors explain similar amounts of variance in the data, and the respective eigenvalues have similar small values [blue disks in Fig. 6(b)]. Contrary to this, in the right panel of Fig. 6(c) similar "bell" shaped patterns are seen in each $J$ segment of $\mathbf{e}_{1}^{*}$, which represents almost five times the variance of the data $\left(\lambda_{1}^{*} \approx 0.25\right)$ as compared with $\mathbf{e}_{2}^{*}\left(\lambda_{2}^{*} \approx 0.05\right)$, resulting in a large gap seen in the $\lambda_{k}^{*}$ spectrum [see orange triangles in Fig. 6(b)]. A sharp global pattern is also seen in $\mathbf{e}_{2}^{*}$, but no clear visible gap in the $\lambda_{k}^{*}$ spectrum is seen for $k \geq 2$.

Given the results above, one way of focusing on the collective dynamics rather than on a detailed view of PS cluster formation, is to monitor the leading $\lambda_{1}^{*}$, that corresponds to the stronger, global, dynamical feature present in the data. This is shown in Fig. 7(a) for increasing values of the coupling gain $g$. As a crosscheck, the svM-SSA results were compared with two order parameters that have been proposed to quantify the level of synchronism: (i) the mean square deviation ${ }^{24} \mathcal{K}=\left\langle V(t)-\langle V(t)\rangle^{2}\right\rangle$, where $\langle\cdot\rangle$ stands for time average and $V(t)=\sum_{j=1}^{N} x_{j}(t)$ is the local field potential (LFP); and (ii) the spike distance metric $D .{ }^{25,26}$ As seen in Fig. 7(a), the overall behavior of $\lambda_{1}^{*}(g), \mathcal{K}(g)$ and $1-$ $D$ is similar. Therefore, in the context of a large network and with just the raster time series of spike events, the svM-SSA is able to provide the general picture of PS.

\section{CONCLUSIONS}

In most studies involving neuron models, it is common to use the first state variable- the membrane potential—for 

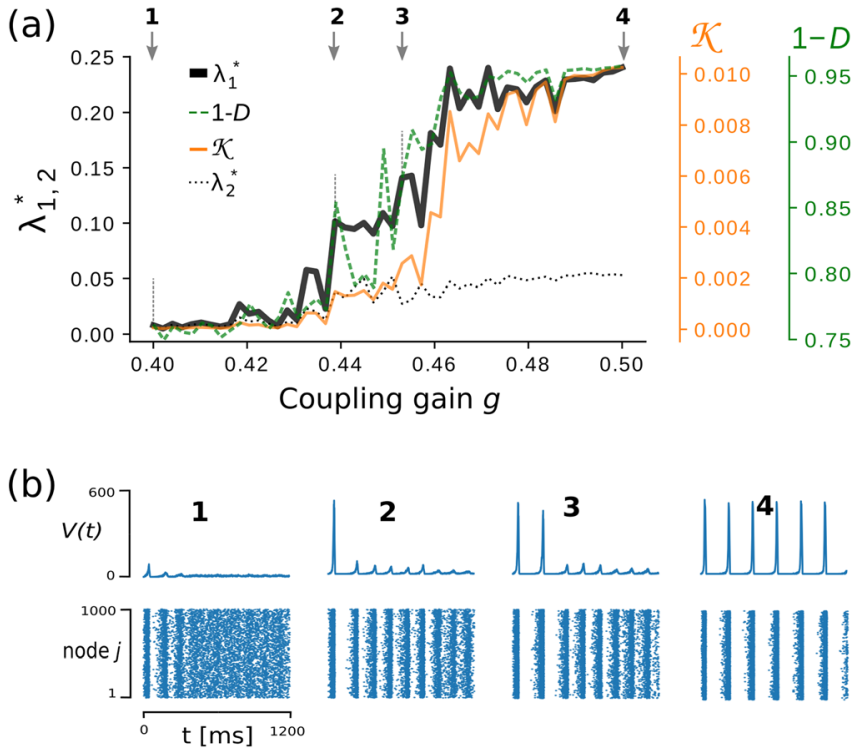

FIG. 7. Synchronization analysis for the $N=1000$ pulse-coupled Izhikevich neuron models. (a) The two black (thick and dashed) lines correspond to the highest singular values, $\lambda_{1,2}^{*}$, the orange (thin) one to the order parameter $\mathcal{K}$ and the green (dashed) one is the order parameter $1-D$. (b) Local field potential $V(t)$ and the corresponding signal raster plot at values of $g$ marked by arrows and dashed lines in (a).

monitoring or controlling purposes. This choice of variable results from the fact that, in experimental neuron networks, only this variable can be actually measured. However, it is important, not only from a theoretical point of view, to know if such a choice is the most adequate in terms of the dynamical behavior. In practice, the analysis of a variable that conveys poor dynamical information could imply unreliable and wrong results. ${ }^{3,35,37}$

One of the objectives of this paper has been to investigate the observability properties of neuron models. This has been done using three different quantifiers for observability: coefficients determined numerically from the model equations, ${ }^{29}$ from data ${ }^{3}$ or symbolic coefficients analytically obtained from the model equations. ${ }^{28}$ This procedure turned out to reveal the limitations of some techniques, for instance, due to the complexity of the equations and the physical interpretation of the variables, investigating the observability of the Hodgkin-Huxley model is viable only using the dataestimated coefficients or the symbolic observability coefficients. Also, the performance of such a method using discontinuous data as for the Izhikevich's spiking neuron model is uncertain. This mostly results from the fact that in this latter model, the switching mechanism is not fully described by the equations and there is at least one missing variable in the model for having a complete description of the underlying mechanisms. Hence observability was investigated from a truncated model and it remains an open question how to proceed with such discontinuous systems.

In summary the variables that convey greater observability were: the membrane potential in the Hodgkin-Huxley and Izhikevich's models (especially during chattering), whereas for FitzHugh-Nagumo the observability provided by the potential and recovery variables is comparable. The Hindmarsh-Rose model has some peculiarities in what concerns observability. The membrane potential and fast recovery variable reveal the fast time scales such as the spikes in the chattering regime, whereas the $z$ variable (slow recovery) is the only one to clearly reveal the chaotic nature of the dynamics when it occurs.

Some of the aforementioned models were investigated in the context of synchronization. In particular, networks formed of five phase coherent FitzHugh-Nagumo neurons, five bursting Hindmarsh-Rose neurons (not shown), and one with 1000 Izhikevich neurons were analyzed. A technique known as structured-varimax multivariate singular spectrum analysis (sv-MSSA), from a variable that provides good observability of the dynamics, was used to successfully detect phase synchronization in the networks. The results attained with the sv-MSSA were crosschecked with more conventional procedures such as the estimation of mean frequencies and instantaneous phases, the use of spatiotemporal patterns, for the example with FitzHugh-Nagumo models; and local field potential, its mean square deviation, the spike distance metric and signal raster plots, for the example with 1000 Izhikevich neurons. Two interesting features of this technique is that it does not require computing the phase and it is able to detect synchronization in situations where other methods like the computation of mean frequencies or visual inspection of spatioteporal patterns give an unclear indication. It is therefore believed that the field of neuroscience would profit from such a tool.

\section{ACKNOWLEDGMENTS}

The authors gratefully acknowledge financial support from the Conselho Nacional de Desenvolvimento Científico e Tecnológico (CNPq), Brazil.

${ }^{1}$ L. A. Aguirre, "A nonlinear correlation function for selecting the delay time in dynamical reconstructions," Phys. Lett. 203A(2-3), 88-94 (1995).

${ }^{2}$ L. A. Aguirre and C. Letellier, "Observability of multivariable differential embeddings,” J. Phys. A: Math. Gen. 38, 6311-6326 (2005).

${ }^{3}$ L. A. Aguirre and C. Letellier, "Investigating observability properties from data in nonlinear dynamics," Phys. Rev. E 83, 066209 (2011).

${ }^{4}$ L. A. Aguirre and C. Letellier, "Controllability and synchronizability: Are they related?," Chaos, Solitons Fractals 83, 242-251 (2016).

${ }^{5}$ I. Belykh, E. De Lange, and M. Hasler, "Synchronization of bursting neurons: What matters in the network topology," Phys. Rev. Lett. 94, 188101 (2005).

${ }^{6}$ E. Bianco-Martinez, M. S. Baptista, and C. Letellier, "Symbolic computations of nonlinear observability," Phys. Rev. E 91, 062912 (2015).

${ }^{7}$ D. S. Broomhead and G. P. King, "Extracting qualitative dynamics from experimental data," Physica D 20, 217-236 (1986).

${ }^{8} \mathrm{~J}$. W. Brown, "The tale of the neuroscientist and the computer: Why mechanistic theory matters," Front. Neurosci. 8, 349 (2014).

${ }^{9}$ R. FitzHugh, "Impulses and physiological states in theoretical mmodel of nerve membrane," Biophys. J. 1, 445-466 (1961).

${ }^{10} \mathrm{~B}$. Friedland, "Controllability index based on conditioning number," J. Dyn. Syst., Meas. Control 97(4), 444-445 (1975).

${ }^{11}$ M. Frunzete, J. P. Barbot, and C. Letellier, "Influence of the singular manifold of nonobservable states in reconstructing chaotic attractors," Phys. Rev. E 86(2), 026205 (2012).

${ }^{12}$ M. Girardi-Schappo, M. H. R. Tragtenberg, and O. Kinouchi, "A brief history of excitable map-based neurons and neural networks," J. Neurosci. Methods 220, 116-130 (2013).

${ }^{13}$ A. Groth, "Monte Carlo singular spectrum analysis (SSA) revisited: Detecting oscillator clusters in multivariate datasets," J. Clim. 28(19), 7873-7893 (2015).

${ }^{14} \mathrm{~A}$. Groth and M. Ghil, "Multivariate singular spectrum analysis and the road to phase synchronization,” Phys. Rev. E 84, 036206 (2011). 
${ }^{15}$ M. Ghil, M. R. Allen, M. D. Dettinger, K. Ide, D. Kondrashov, M. E. Mann, and P. Yiou, "Advanced spectral methods for climate time series," Rev. Geophys. 40(1), 3.1-3.41, doi:10.1029/2000RG000092 (2002).

${ }^{16}$ R. Hermann and A. Krener, "Nonlinear controllability and observability," IEEE Trans. Autom. Control 22(5), 728-740 (1977).

${ }^{17}$ J. L. Hindmarsh and R. M. Rose, "A model of neuronal bursting using three coupled first order differential equations," Proc. R. Soc. London B 221, 87-102 (1984).

${ }^{18}$ A. L. Hodgkin and A. F. Huxley, "A quantitative description of membrane current and its application to conduction and excitation in nerve," J. Physiol. 117, 500-544 (1952).

${ }^{19}$ A. Isidori, Nonlinear Control Systems (Springer, London, 1995).

${ }^{20}$ E. M. Izhikevich, "Simple model of spiking neurons," IEEE Trans. Neural Networks 14(6), 1569-1572 (2003).

${ }^{21} \mathrm{E}$. M. Izhikevich, "Which model to use for cortical spiking neurons?," IEEE Trans. Neural Networks 15(5), 1063-1070 (2004).

${ }^{22} \mathrm{E}$. M. Izhikevich and G. M. Edelman, "Large-scale model of mammalian thalamocortical systems," PNAS 105(9), 3593-3598 (2008).

${ }^{23}$ R. E. Kalman, "On the general theory of control systems," in Proceedings of the First IFAC Congress Automatic Control (Butterworths, London, 1960), pp. 481-492.

${ }^{24}$ S.-Y. Y. Kim and W. Lim, "Coupling-induced population synchronization in an excitatory population of subthreshold Izhikevich neurons," Cognit. Neurodyn. 7(6), 495-503 (2013).

${ }^{25}$ T. Kreuz, D. Chicharro, M. Greschner, and R. G. Andrzejak, "Timeresolved and time-scale adaptive measures of spike train synchrony," J. Neurosci. Methods 195(1), 92-106 (2011).

${ }^{26}$ T. Kreuz, D. Chicharro, C. Houghton, R. G. Andrzejak, and F. Mormann, "Monitoring spike train synchrony," J. Neurophysiol. 109(5), 1457-1472 (2013).

${ }^{27}$ C. Letellier and L. A. Aguirre, "Investigating nonlinear dynamics from time series: The influence of symmetries and the choice of observables," Chaos 12(3), 549-558 (2002).

${ }^{28}$ C. Letellier and L. A. Aguirre, "Symbolic observability coefficients for univariate and multivariate analysis," Phys. Rev. E 79, 066210 (2009).

${ }^{29}$ C. Letellier, L. A. Aguirre, and J. Maquet, "Relation between observability and differential embeddings for nonlinear dynamics," Phys. Rev. E 71, 066213 (2005).

${ }^{30}$ C. Letellier, J. Maquet, L. Le Sceller, G. Gouesbet, and L. A. Aguirre, "On the non-equivalence of observables in phase-space reconstructions from recorded time series,” J. Phys. A 31, 7913-7927 (1998).
${ }^{31}$ C. Letellier, I. Sendiña-Nadal, E. Bianco-Martinez, and M. S. Baptista, “A symbolic network-based nonlinear theory for dynamical systems observability," preprint (2017).

${ }^{32}$ E. Lowet, M. J. Roberts, P. Bonizzi, J. Karel, and P. De Weerd, "Quantifying neural oscillatory synchronization: A comparison between spectral coherence and phase-locking value approaches," PLoS One 11, e0146443 (2016).

${ }^{33}$ S. Mangiarotti and C. Letellier, "Topological analysis for designing a suspension of the Hnon map," Phys. Lett. A 379, 3069-3074 (2015).

${ }^{34}$ J. Nagumo, S. Arimoto, and S. Yoshizawa, "An active pulse transmission line simulating nerve axon,” Proc. IRE 50(10), 2061-2070 (1962).

${ }^{35}$ L. L. Portes and L. A. Aguirre, "Enhancing multivariate singular spectrum analysis for phase synchronization: The role of observability," Chaos 26, 093112 (2016a).

${ }^{36}$ L. L. Portes and L. A. Aguirre, "Matrix formulation and singular-value decomposition algorithm for structured varimax rotation in multivariate singular spectrum analysis," Phys. Rev. E 93, 052216 (2016b).

${ }^{37}$ L. L. Portes, R. N. Benda, H. Ugrinowitsch, and L. A. Aguirre, "Impact of the recorded variable on recurrence quantification analysis of flows," Phys. Lett. A 378(32-33), 2382-2388 (2014).

${ }^{38}$ O. E. Rössler, “An equation for continuous chaos," Phys. Lett. A 57, 397-398 (1976).

${ }^{39}$ S. J. Schiff, Neural Control Engineering (The MIT Press, Cambridge, Massachusetts, 2012).

${ }^{40}$ M. Sedigh-Sarvestani, S. J. Schiff, and B. J. Gluckman, "Reconstructing mammalian sleep dynamics with data assimilation," PLoS Comput. Biol. 8(11), e1002788 (2012).

${ }^{41}$ I. Sendiña-Nadal, S. Boccaletti, and C. Letellier, "Observability coefficients for predicting the class of synchronizability from the algebraic structure of the local oscillators," Phys. Rev. E 94, 042205 (2016).

${ }^{42}$ C. Siettos and J. Starke, "Multiscale modeling of brain dynamics: From single neurons and networks to mathematical tools," WIREs Syst. Biol. Med. 8, 438-458 (2016).

${ }^{43}$ F. Su, J. Wang, H. Li, B. Deng, H. Yu, and C. Liu, "Analysis and application of neuronal network controllability and observability," Chaos 27, 023103 (2017).

${ }^{44}$ A. J. Whalen, S. N. Brennan, T. D. Sauer, and S. J. Schiff, "Observability and controllability of nonlinear networks: The role of symmetry," Phys. Rev. X 5, 011005 (2015).

${ }^{45}$ H. U. Voos, J. Timmer, and J. Kurths, "Nonlinear dynamical system identification from uncertain and indirect measurements," Int. J. Bifurcation Chaos 14(6), 1905-1933 (2004). 